\title{
Large-eddy simulations of round jets at a Mach number of 0.9 impinging on a plate with and without a hole
}

\author{
Mathieu Varé* and Christophe Bogey ${ }^{\dagger}$ \\ Univ Lyon, École Centrale de Lyon, INSA Lyon, Université Claude Bernard Lyon I, CNRS \\ Laboratoire de Mécanique des Fluides et d'Acoustique, UMR 5509 \\ F-69134 Ecully, France
}

\begin{abstract}
Four initially highly disturbed round jets at a Mach number of 0.9 impinging on a plate located at a distance $L=6 r_{0}$, where $r_{0}$ is the nozzle radius, have been computed by LargeEddy Simulation (LES). Three of the plates have a hole of diameter $d=2 r_{0}, 3 r_{0}$ and $4.4 r_{0}$, centered on the jet axis, whereas the fourth one has no hole, in order to study the effects of the hole diameter on the jet flow and acoustic fields. For the cases with no hole and the holes of diameter $d=2 r_{0}$ and $3 r_{0}$, a feedback loop establishes between the jet nozzle and the plate, which generates intense tones in the acoustic spectra. For $\boldsymbol{d}=\mathbf{4 . 4 r _ { 0 }}$, a similar feedback loop is observed, but it generates tones of weaker amplitude. For all cases, the feedback frequency is in agreement with the classical model of an aeroacoustic feedback between the nozzle and the plate. The acoustic levels are however reduced when the hole diameter increases. The same standing wave pattern between the nozzle and the plate is highlighted by a study of the aerodynamic field at the main tonal frequency. Moreover, for $d=2 r_{0}$ and $3 r_{0}$, flow rate fluctuations are caused by turbulent structures passing through the hole at the feedback frequency, which creates a tonal radiation downstream of the plate. A model of this radiation by a monopole source of equivalent flow rate is proposed.
\end{abstract}

\section{Introduction}

Intense acoustic tones are generated by the impingement of jets on a plate. These tones were observed experimentally for high subsonic jets by many researchers, including Powell [1], Ho and Nosseir [2], Nosseir and Ho [3], Neuwerth [4] or Preisser [5]. In these studies, a staging phenomemon of the main tone frequency was measured when the nozzle-to-plate distance increases, which has led Powell [1] to explain the tone generation by a feedback mechanism between turbulent structures convected downstream from the nozzle to the plate and acoustic waves propagating upstream from the plate to the nozzle. This feedback loop was noticed in supersonic jet impingement as well. For ideally expanded supersonic jets, the establishment of this loop has been highlighted experimentally by Norum [6] and numerically by Gojon $e t$ al. [7] and Bogey \& Gojon [8]. For underexpanded jets, it has been visualized in the experimental work of Henderson $e t$ al. [9], Buchmann et al. [10], Risborg \& Soria [11], and Mitchell et al. [12] and in the numerical simulations of Gojon \& Bogey [13] and Dauptain et al. [14]. In particular, a standing wave pattern in the jet has been exhibited by applying a Fourier decomposition to the pressure field at the main tonal frequency by Gojon et al. [7].

Jets impinging on a plate with a hole have been the subject of few studies. For subsonic jets, tones similar to those generated by jets impinging on a plate with no hole have been first observed by Sondhauss [17] and Rayleigh [18], and they have been refered to as hole tones. As for the plate with no hole, they are generated by a feedback mechanism between the hole edges and the nozzle. Indeed, the same staging phenomenon of the tone frequency as the nozzle-to-plate distance increases was observed in several experiments, such as those of Chanaud \& Powell [19] and Vinoth \& Rathakrishnan [20]. Hole tone generation has been studied over a wide range of Mach numbers, numerically for very low Mach numbers by Langthjem \& Nakano [21] and Matsuura \& Nakano [22], and experimentally for high subsonic and supersonic jets by Umeda et al. [23] and Umeda \& Ishii [24]. Umeda et al. [23] have notably visualized the feedback loop between the hole edges and the nozzle for jets at a Mach number of 0.94 and 1.54 using shadowgraphy. They also observed a tonal radiation at the feedback frequency downstream of the plate. However, no investigation was focused on this downstream radiation or on the effects of the hole on the feedback properties, namely the frequency and intensity of the tones. More recently, jets impinging on a plate with a hole have been studied for a Mach number of 3.7

\footnotetext{
*PhD student, mathieu.vare @ec-lyon.fr

†CNRS Research Scientist, AIAA Senior Member \& Associate Fellow, christophe.bogey@ec-lyon.fr
} 
by Tsutsumi et al. [15] and for a Mach number of 3.1 by Troyes et al. [16]. No feedback loop was observed for these very high Mach numbers.

In the present work, Large-Eddy Simulations (LES) of four subsonic round jets impinging on a plate with and without a hole are carried out in order to investigate the influence of the hole diameter on the feedback mechanism establishing between the nozzle lips and the hole edges. The jets are at a Mach number of 0.9 and a Reynolds number of $10^{5}$, and are initially highly disturbed. They impinge on a plate located at a distance $L=6 r_{0}$ from the nozzle exit, where $r_{0}$ is the jet radius. Three of the plates have a hole of diameter $d=2 r_{0}, 3 r_{0}$ and $4.4 r_{0}$ whereas the last one has no hole. The jet flow and acoustic fields are presented with the aim of visualizing the feedback loop. Pressure spectra are examined in order to highlight the possible tonal frequencies. The azimuthal structure of the jets is also investigated. For each tone frequency, a Fourier decomposition of the pressure field is applied to reveal the standing wave patterns and oscillation modes of the jet. Finally, the acoustic radiation downstream of the plate is modeled by a monopole source, whose frequency is the same as the jet flow rate through the hole.

The paper is organized as follows. The jet parameters and numerical methods used in the LES are documented in section [II The flow and acoustic properties of the four jets are then described in section III Concluding remarks are given in section $\mathrm{IV}$.

\section{Parameters}

\section{A. Jet parameters}

The four jets have a Mach number $M=u_{j} / c_{0}$ of 0.9 and a Reynolds number $R e_{D}=u_{j} D / v$ of $10^{5}$, where $u_{j}$ is the jet velocity, $D$ the nozzle diameter and $v$ the air kinematic viscosity. They are exhausted by a cylindrical nozzle of radius $r_{0}$. The jets are at ambient temperature and pressure $T_{0}=293 \mathrm{~K}$ and $p_{0}=10^{5} \mathrm{~Pa}$. In the nozzle, a Blasius boundary-layer mean velocity profile is imposed with a boundary-layer thickness of $\delta=0.15 r_{0}$. Vortex rings non-correlated in the azimuthal direction are added in the boundary-layer at $z=-r_{0}$ to obtain a turbulent intensity peak of $9 \%$ at the nozzle exit, as represented in figure 1. This excitation is the same as in the simulations of a free jet at $M=0.9$ of Bogey [25]. The jets impinge on a plate located $L=6 r_{0}$ downstream of the nozzle exit, with a width of $e=0.4 r_{0}$. In one case, the plate has no hole whereas for three other ones the plate has a hole of diameter $d=2 r_{0}, 3 r_{0}$, and $4.4 r_{0}$. The four cases are referred to as jetd0, jetd2, jetd 3 and jetd4 respectively. The nozzle-to-plate distance, the plate width and the diameters of the hole are the same as in the experiments of Umeda et al. [23].

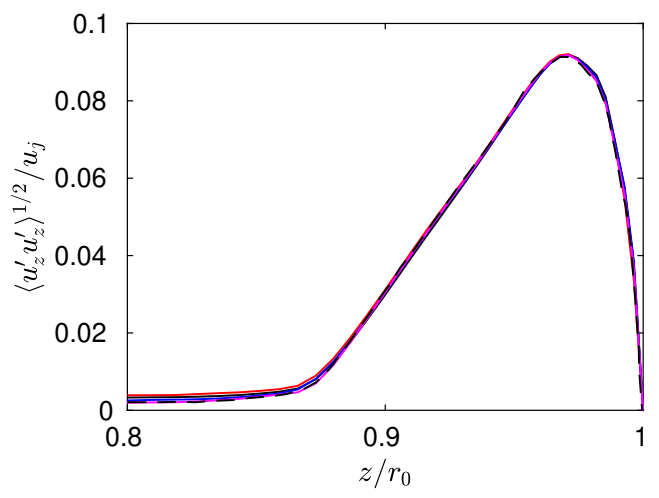

Fig. 1 Nozzle-exit radial profiles of axial turbulence intensity $\left\langle u_{z}^{\prime} u_{z}^{\prime}\right\rangle^{1 / 2} / u_{j}:-$ jetd0, - jetd2, - jetd3, jetd4 and - - - free jet [25].

\section{B. Numerical parameters}

In the simulations, the unsteady compressible Navier-Stokes equations are solved in cylindrical coordinates $(r, \theta, z)$ using an OpenMP based in-house solver. The time integration is performed using a six-stage Runge-Kutta algorithm [26] and the spatial derivatives are evaluated with an eleven-point low-dissipation and low-dispersion finite-difference scheme [27]. At the end of each time step, a selective filtering is applied to remove grid-to-grid oscillations [26]. This 
filter enables to dissipate kinetic turbulent energy near the grid cut-off frequency, as a subgrid-scale model. Solid and adiabatic wall conditions are imposed to the nozzle and plate walls. The radiation boundary conditions of Tam $\&$ Dong [28] are implemented at the lateral and radial boundaries of the computational domain. They are combined with sponge zones using grid stretching and Laplacian filtering to avoid significant numerical reflections [29]. The singularity at $r=0$ is treated by applying the method developped by Mohseni \& Colonius [30]. The first point close to the axis is thus located at $r=\Delta r / 2$, where $\Delta r$ is the radial mesh size near the jet axis. The effective azimuthal resolution near the origin of the polar coordinates is reduced down to $2 \pi / 16$ in order to increase the admissible time step of the simulation [31].

\section{Computational parameters}

The mesh grids used for the four simulations contain between 540 millions and 1.4 billions of points, as reported in table 1. It extends out to $r=15 r_{0}$ in the radial direction and down to $z=6 r_{0}$ and $z=40 r_{0}$ in the axial direction for the full and perforated plates, respectively. The radial mesh spacing, represented in figure 2 a), is equal to $\Delta r=0.014 r_{0}$ on the jet centerline and progressively decreases down to $\Delta r=0.0036 r_{0}$ at $r=r_{0}$, in the shear layers. It then increases to reach a maximum value of $\Delta r_{\max }=0.075 r_{0}$ for $r>6.2 r_{0}$, which allows us to obtain a Strouhal number $S t=f D / u_{j}$ of 5.9 for an acoustic wave with five points per wavelength. In the azimuthal direction, the grid is uniform and $n_{\theta}=1024$ points are used. The axial mesh spacing $\Delta z$, presented in figure 2 (b), is minimal and is equal to $\Delta z=0.0072 r_{0}$ at the nozzle exit. It increases and reaches $\Delta z=0.012 r_{0}$ between $r=2 r_{0}$ and $r=4 r_{0}$. Farther downstream, the axial mesh spacing is then reduced to be again equal to $\Delta z=0.0072 r_{0}$ near the plate, at $z=6 r_{0}$. After the plate, it increases gradually and reaches $\Delta z_{\max }=0.05 r_{0}$ at $z=40 r_{0}$. The extrema values of the mesh spacing and the stretching rates in both axial and radial directions are the same as in the study of Bogey [25], where grid convergency study was performed for the free jet with the same ejection conditions. The simulation time is of $500 r_{0} / u_{j}$ for jetd $0,410 r_{0} / u_{j}$ for jetd 2 , $365 r_{0} / u_{j}$ for jetd 3 and $425 r_{0} / u_{j}$ for jetd 4 .

\begin{tabular}{ccccc}
\hline & $n_{r}$ & $n_{\theta}$ & $n_{z}$ & $n_{r} \times n_{\theta} \times n_{z}$ \\
\hline jetd0 & 559 & 1024 & 940 & $5.4 \times 10^{8}$ \\
jetd2, jetd3, jetd4 & 559 & 1024 & 2430 & $1.4 \times 10^{9}$ \\
\hline
\end{tabular}

Table 1 Mesh parameters: numbers of points $n_{r}, n_{\theta}$ and $n_{z}$ in the radial, azimuthal and axial directions, and total number of points.
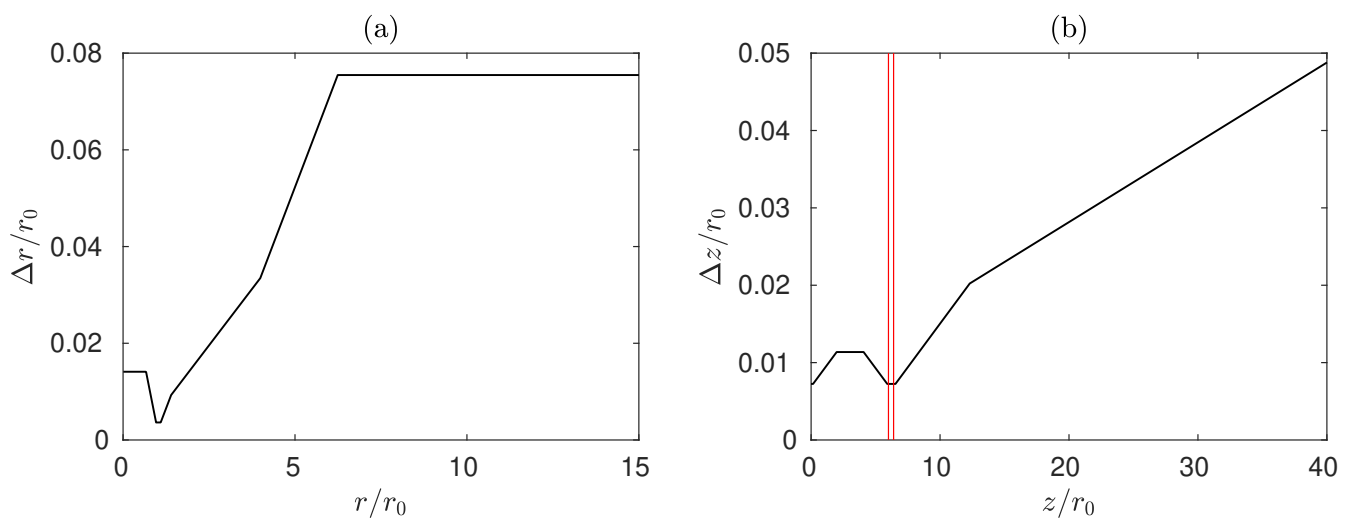

Fig. 2 Variations of (a) radial and (b) axial mesh spacings; — positions of the upstream and downstream faces of the plate. 


\section{Results}

\section{A. Snapshots of the flow and acoustic fields}

Snapshots of the jet flow and acoustic fields are given in figure 3. where the vorticity norm is shown inside the flow, and pressure fluctuations outside. All jets are delimited by thin layers of vorticity near the nozzle. The mixing layers thicken with the axial distance because of the development of vortical structures. For jetd0, jetd2 and jetd3, presented in figures $3(a, b, c)$, these structures impinge on the plate, creating a wall jet. For the plate with no hole and the plates with holes of diameter $d=2 r_{0}$ and $3 r_{0}$, the flow field is dominated by large distorted vortical structures near the impingement region, but not for the plate with a hole of diameter $d=4.4 r_{0}$. For jetd 2 , jetd 3 and jetd 4 , after crossing the plate, the shear layers develop until they merge, closing the potential core of the jets around $z=15 r_{0}$.

For jetd0, jetd2 and jetd3, the impingement of the jet turbulent structures on the plate or on the hole edges generates strong acoustic waves in the pressure fields. Their amplitudes are around $1000 \mathrm{~Pa}$, which is much higher than the amplitude obtained in the pressure field of the free jet [25], which is typically of $50 \mathrm{~Pa}$. Moreover, the magnitude of the pressure fluctuations decreases slightly when the hole diameter increases. For jetd 2 and jetd3, the periodically separated wavefronts of the pressure waves indicates a tonal radiation upstream of the plate, but also downstream. For jetd4, no clear organization of the acoustic field is visible, contrary to the other cases. The amplitude of the pressure fluctuations is twice as small as for the other jets.

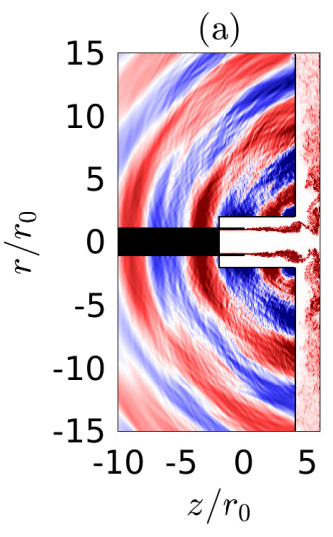

(c)

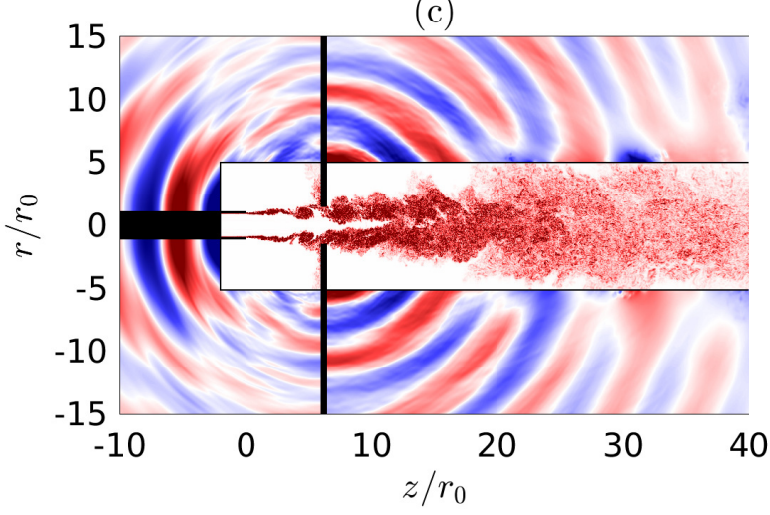

(b)

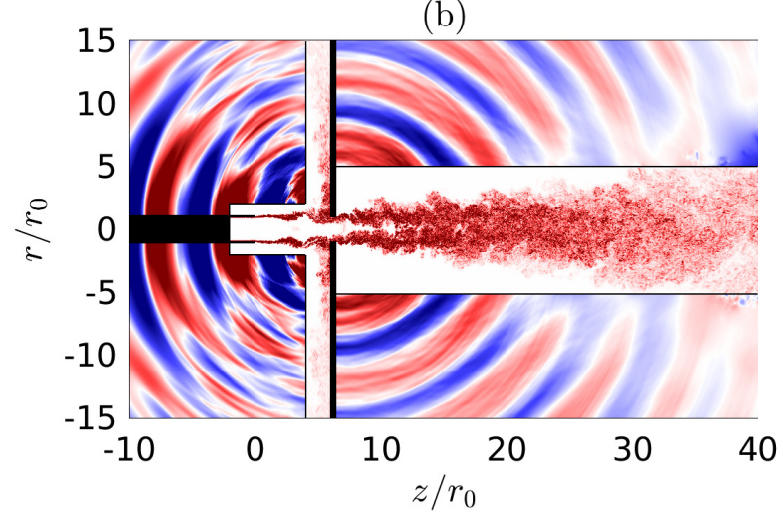

(d)

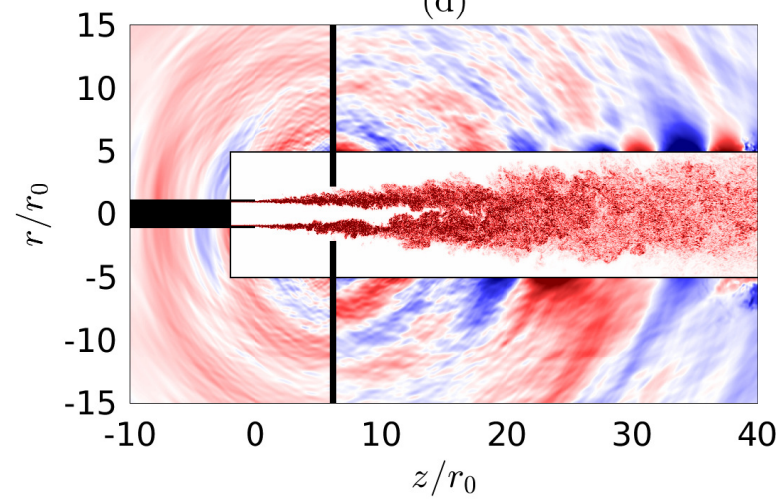

Fig. 3 Snapshots in the $(z, r)$ plane of the vorticity norm in the flow and of the fluctuating pressure outside for (a) jetd0, (b) jetd2, (c) jetd3 and (d) jetd4. The color scales range from 0 up to $7 u_{j} / r_{0}$ for the vorticity norm, from white to red, and between $(a, b, c) \pm 0.01 p_{0}$ and (d) $\pm 0.005 p_{0}$ for the pressure, from blue to red. 


\section{B. Flow field properties}

The mean density fields of the four jets are represented in figure 4. For all jets, the density is the lowest in the shear layers. Except for jetd4, compression cells are present inside the jet. For jetd0, the air inside the jet is progressively compressed down to $z=2 r_{0}$. A second compression cell is present between $z=3 r_{0}$ and $z=4 r_{0}$. Finally, the air undergoes an intense compression from $z=4.5 r_{0}$ to the plate. This high density zone corresponds to the impingement area and extends up to $r=1.8 r_{0}$ on the plate in the radial direction. For jetd2, the density patterns are similar to those for the plate with no hole. Three compression zones upstream of the impingement zone appear in figure $4 \mathrm{~b}$ ). They are located from $z=r_{0}$ to $z=2.6 r_{0}$, from $z=3 r_{0}$ to $z=4.1 r_{0}$ and from $z=4.7 r_{0}$ to $z=5.4 r_{0}$. Moreover, the density in these zones is stronger than for jetd0, which causes a slight radial expansion of the jet from $z=3 r_{0}$ to $z=4.5 r_{0}$. The density is the highest near the hole, between $z=5.7 r_{0}$ and $z=6.4 r_{0}$. The impingement area is smaller than for Jetd 0 and has a radial extension of $1.4 r_{0}$. Downstream of the plate, the density in the potential core is constant and equal to the ambient density. The density field of jetd3, presented in figure 4(c), is close to that of jetd2. Upstream of the plate, three lobes of high density are found in the jet between $z=0.7 r_{0}$ and $z=2.5 r_{0}, z=2.8 r_{0}$ and $z=4.6 r_{0}$ and $z=4.8 r_{0}$ and $z=5.7 r_{0}$. The densities in these compression areas are slighly lower than for jetd2. Another zone of compression starts in the hole at $z=6.2 r_{0}$ and ends outside at $z=7 r_{0}$. Its radius is equal to $0.7 r_{0}$. Contrary to the plates with no hole and with the smallest hole, no compression is observed near the plate and the hole edges. Downstream of the plate, the density increases very weakly before returning to the ambient value down to the end of the potential core. Finally, for the plate with the largest hole in figure 4 d d), no variations of density are found in the jet contrary to the other cases. The density in the potential core is equal to the ambient density.
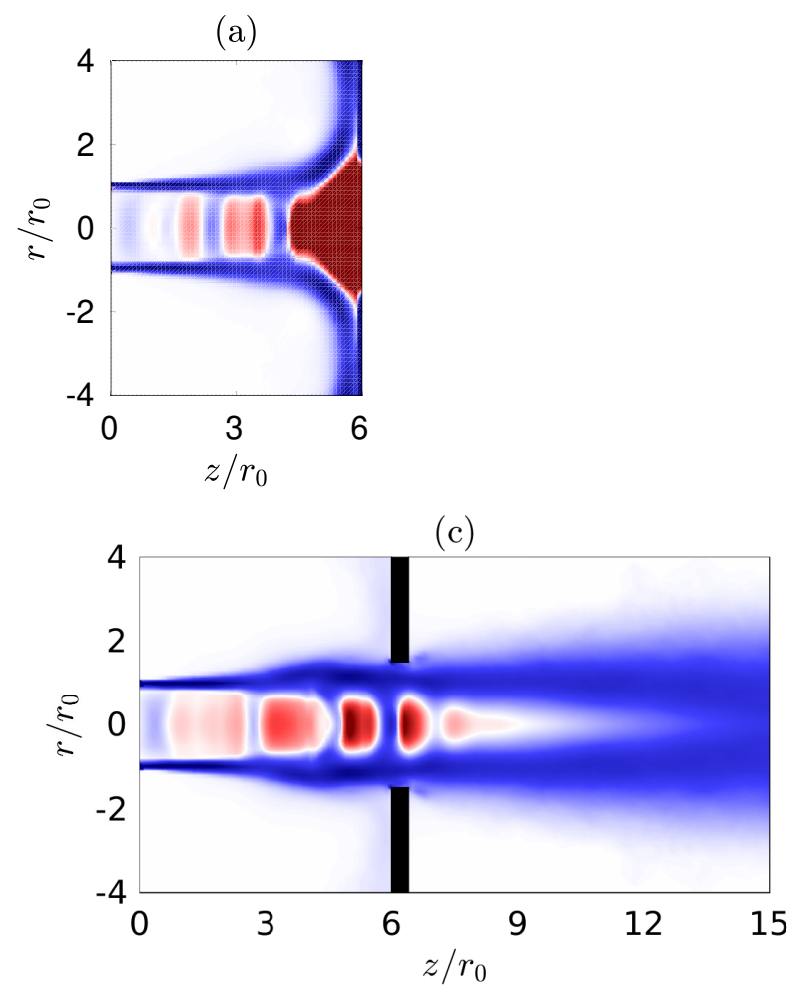

(b)

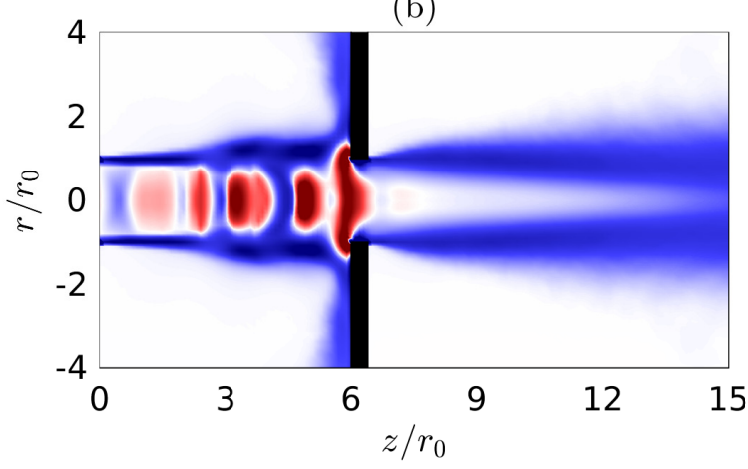

(d)

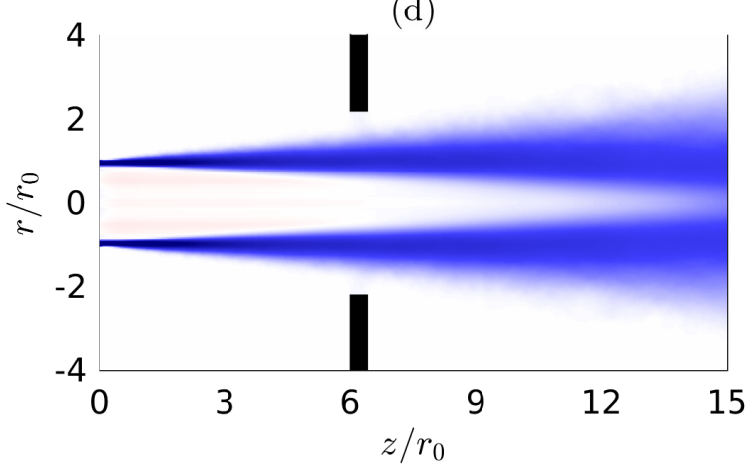

Fig. 4 Mean density fields in the $(z, r)$ plane for (a) jetd0, (b) jetd2, (c) jetd3 and (d) jetd4. The color scales range from $0.95 \rho_{0}$ up to $1.05 \rho_{0}$, from blue to red.

The profiles of centerline mean axial velocity in the impinging jets are presented in figure 5 (a). They are compared to the results for the free jet [25]. Without a hole, the velocity decreases and reaches zero at the plate at $z=6 r_{0}$. For jetd 2 and jetd3, the velocity does not vary much between the nozzle and the jet. Small oscillations are however visible between $z=2 r_{0}$ and $z=6 r_{0}$. They are linked to the compression cells observed in the density fields and generated by 
the impingement on the plate. For jetd4, the velocity remains very close to $u_{j}$ between the nozzle and the plate, as for the free jet.

The variations of the shear-layer momentum thickness with the axial distance are presented in figure 5 b). For jetd4, the shear-layer spreading is the same as for the free jet. The shear-layers of the three other jets develop more rapidly. The jet mixing in these cases is therefore enhanced by the jet impingement on the plates. Note that the mixing layer is thicker for jetd 2 and jetd 3 than for jetd 0 between $z=0$ and $z=4.5 r_{0}$, and the shear-layer of jetd 0 becomes the largest from $z=4.5 r_{0}$ because of the wall jet.

The rms values of axial and radial velocity fluctuations estimated along the nozzle-lip line at $r=r_{0}$ between $z=0$ and $z=6 r_{0}$ are displayed in figure 6. For the impinging jets, they are very similar to those in the free jet down to $z=2 r_{0}$. Farther downstream, the magnitude of the fluctuations for jetd 4 grow continuously and are slightly higher than for the free jet. For the no hole case, they decrease and are equal to zero at the plate at $z=6 r_{0}$. For jetd 2 and jetd 3 , the velocity fluctuations also weaken between $z=2 r_{0}$ and $z=4 r_{0}$, and become stronger between $z=4 r_{0}$ and $z=6 r_{0}$.

(a)

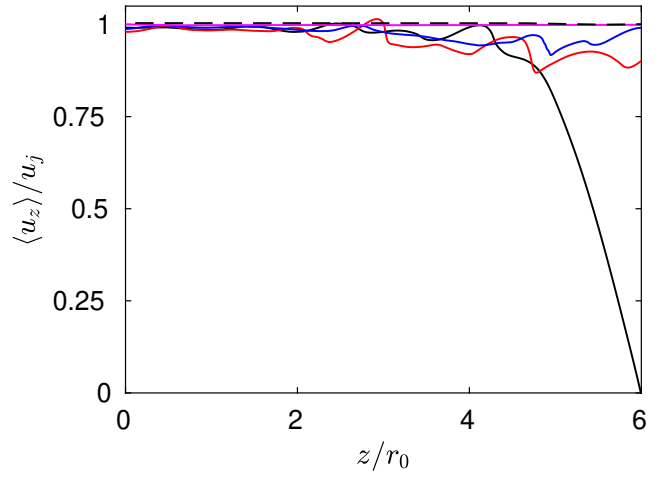

(b)

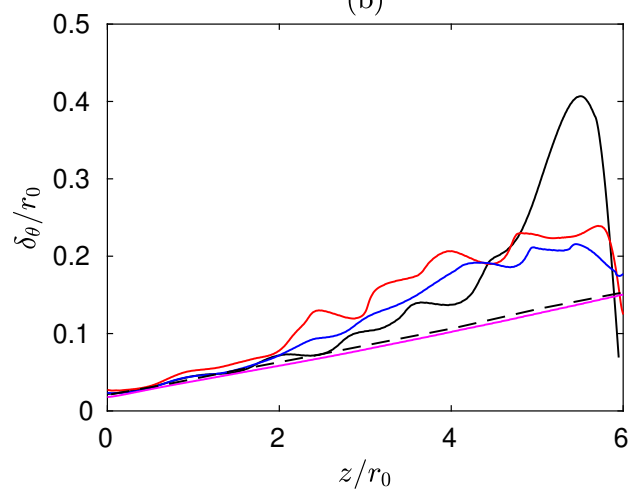

Fig. 5 Variations of (a) mean axial velocity $\left\langle u_{z}\right\rangle / u_{j}$ at $r=0$ and (b) shear-layer momentum thickness $\delta_{\theta} / r_{0}$ : - jetd0, - jetd2, - jetd3, - jetd 4 and - - - free jet [25].

(a)

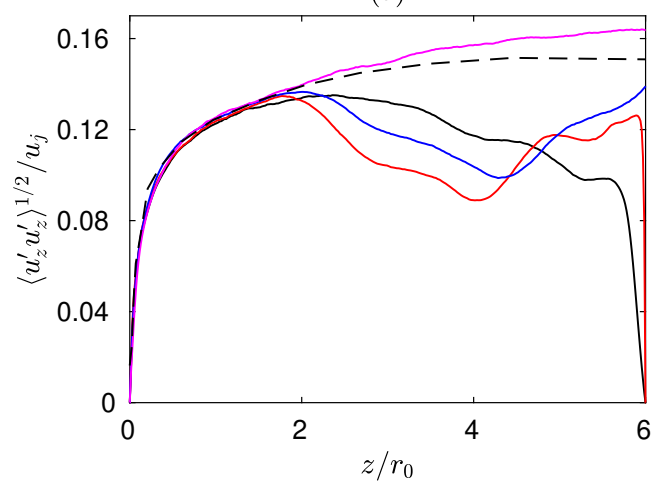

(b)

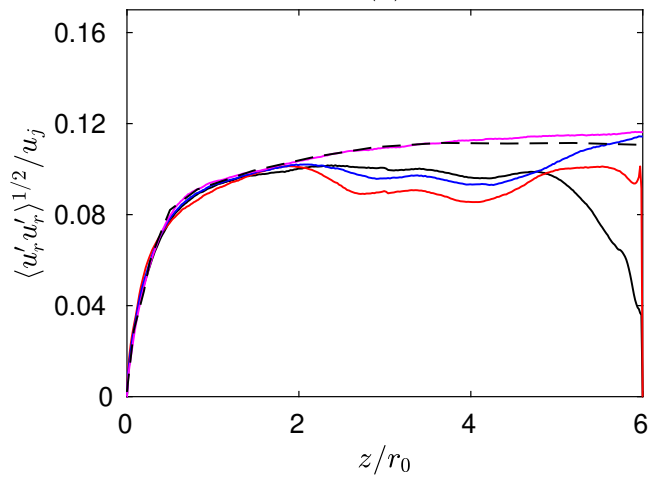

Fig. 6 Variations of (a) axial turbulence intensity $\left\langle u_{z}^{\prime} u_{z}^{\prime}\right\rangle^{1 / 2} / u_{j}$ and (b) radial turbulence intensity $\left\langle u_{r}^{\prime} u_{r}^{\prime}\right\rangle^{1 / 2} / u_{j}$ at $r=r_{0}:$ - jetd $0,-$ jetd2, - jetd3, - jetd 4 and -- free jet.

\section{Convection velocity}

The variations of the convection velocity $u_{c}$ of the shear-layer structures with the axial distance are displayed in figure 7 for the four jets. This velocity has been estimated thanks to cross-correlations in the shear layer at $r=r_{0}$. For jetd0, jetd 2 and jetd3, the convection velocity increases between the nozzle lip and $z \approx 2.5 r_{0}$. Then, it decreases down 
to $z \approx 3.5 r_{0}$. It increases again and reaches a maximum at $z \approx 4.5 r_{0}$. The variations of the convection velocity follow those of the jet centerline velocity, presented in figure $5 \mathrm{la}$ ). For the plate with the largest hole, the convection velocity increases monotonously from a value of $0.5 u_{j}$ at $r=1.5 r_{0}$ up to a value of $0.65 u_{j}$ at $z=6 r_{0}$.

The mean values of the convection velocity $\left\langle u_{c}\right\rangle / u_{j}$ obtained between $z=1.5 r_{0}$ and $z=5 r_{0}$ are $0.64,0.72,0.70$ and 0.60 for jetd 0 , jetd 2 , jetd 3 and jetd4, respectively, which is in agreement with the classical approximation of $2 / 3 u_{j}$. The maximum mean convection velocity is found for jetd 2 whereas the minimum one corresponds to jetd 4 . Moreover, the compression cells observed in the density fields are the strongest for jetd 2 whereas there are none in jetd4, which suggests a link between the strength of these cells and convection velocity oscillations.

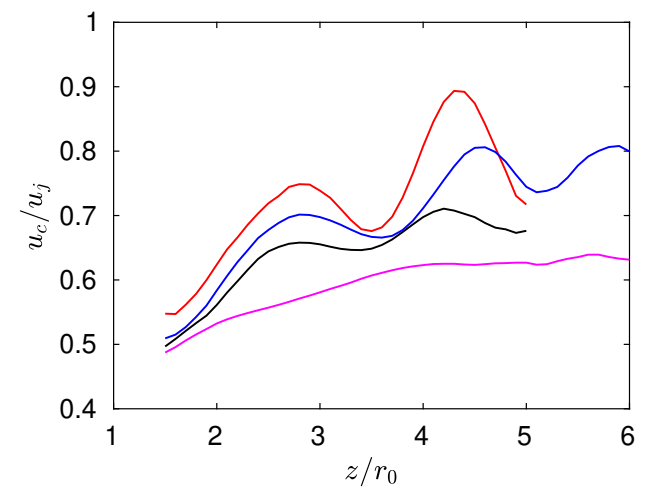

Fig. 7 Convection velocities of the turbulent structures in the shear layers for - jetd0, - jetd2, - jetd 3 and - jetd4.

\section{Overall Sound Pressure Levels}

The Overall Sound Pressure Levels (OASPL) obtained at $r=15 r_{0}$ are shown in figure 8 Upstream of the plate, in figure 8 (a), they are almost constant and around $134 \mathrm{~dB}$ for the case jetd 0 with no hole. For jetd2, the acoustic levels vary as those of jetd0. However, they are reduced of $7 \mathrm{~dB}$ compared to the latter case. Concerning jetd3, between $z=0$ and $z=3 r_{0}$, the OASPL are lower of $6 \mathrm{~dB}$ compared to those of jetd2. Farther downstream, they increase and take the same value as for jetd 2 near to the plate. Nevertheless, this increase is not observed for jetd 0 and jetd2, which might be due to the effects of the wall jets, which are more developed than for jetd3. Finally, the OASPL are the lowest for the plate with the largest hole. They are constant and equal to $107 \mathrm{~dB}$.

The levels downstream of the plate are represented in figure 8 (b). They follow the same evolution for the two plates with the smallest holes. For both jets, they remain close to $130 \mathrm{~dB}$ between the exit of the hole and $z=25 r_{0}$. They decrease from $z=25 r_{0}$ and are equal to $120 \mathrm{~dB}$ at $z=40 r_{0}$. For jetd4, the OASPL are significatively lower with a value of $110 \mathrm{~dB}$. Contrary to the two other jets, no variations of these levels with the axial distance are observed.

The sound levels are represented in figure 9 in order to localize the noise sources. In figure 9 (a), without a hole, the isolines close to the nozzle are almost parallel to the plate. Further from the nozzle in the radial direction, they are distorted and form a lobe. For the plates with a hole represented in figure 9 b), $9(\mathrm{c})$ and 9 d), the isocontours of acoustic levels upstream of the plate form circles centered on the hole in the plate, indicating an acoustic radiation coming from the hole. Contrary to jetd0, no lobe is observed in the isolines. The wall jet on the plate is the most developed for jetd0, which suggests that the distortion of the isolines is due to the wall jet for the case without a hole. Downstream of the plate, for jetd2 and jetd3, the isolines also suggest an acoustic radiation originating from the hole, which is not the case for jetd4.

\section{E. Pressure spectra}

The sound pressure levels obtained at $r=2 r_{0}$ and $z=0$ are plotted in figure 10 as a function of the Strouhal number. For jetd 0 , jetd 2 and jetd 3 , several tones emerge. The dominant tone is located at a frequency of $S t=0.41$ for the three cases and is greater by 25 to $30 \mathrm{~dB}$ than the broadband noise level. Weaker tones are observed at a Strouhal number of $0.68,0.65$ and 0.60 for jetd0, jetd2 and jetd3, respectively. For jetd4, a weak tone is found at a Strouhal number of 0.41. The tones are generated by a feedback mechanism between the nozzle and the plate [1]. This mechanism consists of two 
(a)

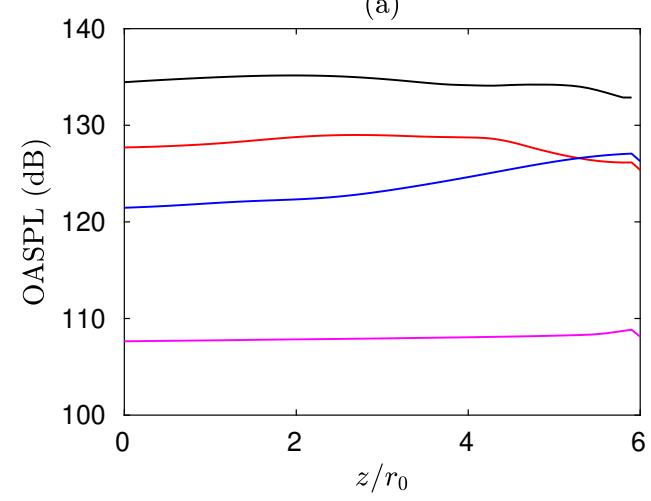

(b)

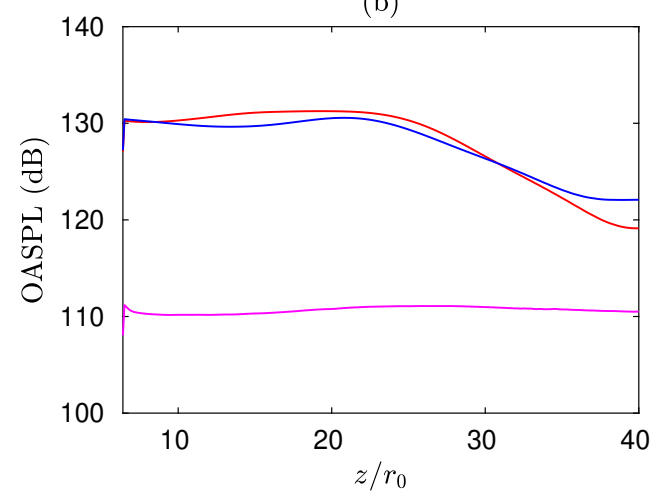

Fig. 8 Variations of OASPL at $r=15 r_{0}$ (a) upstream of the plate and (b) downstream of the plate for - jetd0, — jetd2, — jetd3 and - jetd4.
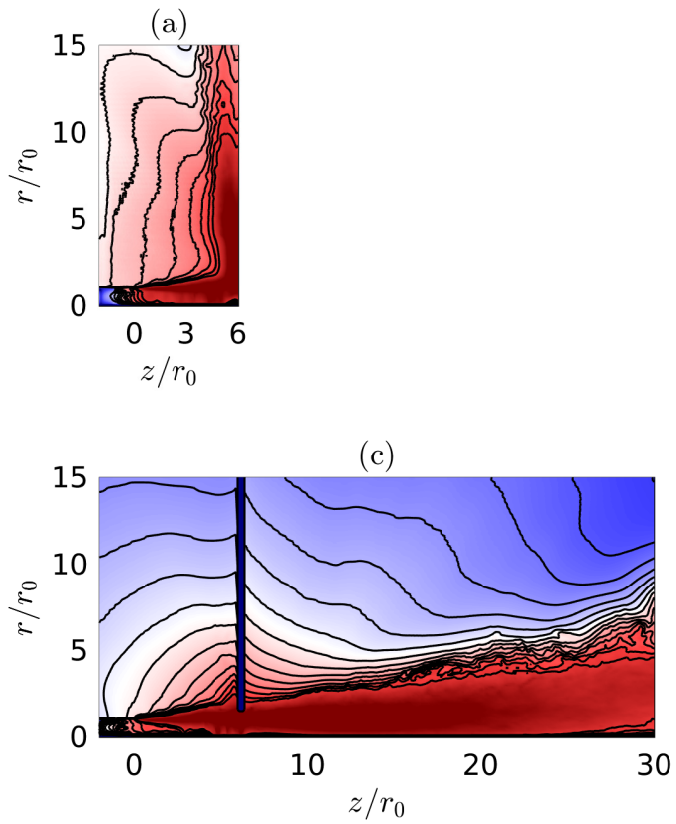

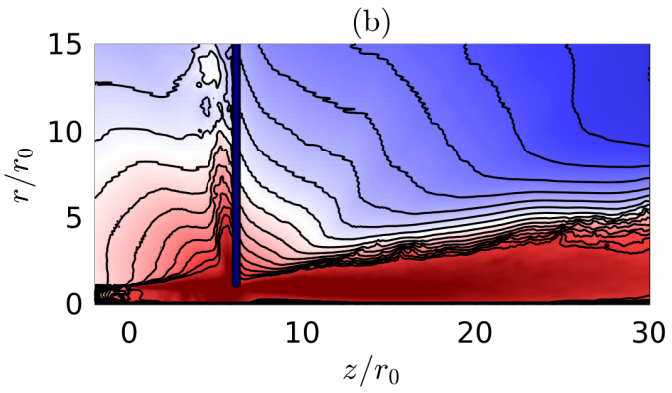

(d)

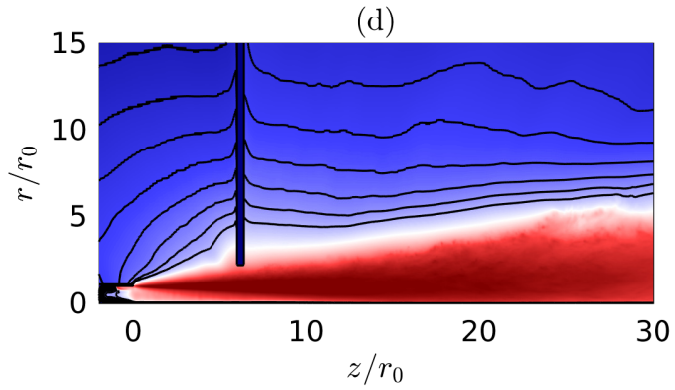

Fig. 9 Representations of the OASPL in the $(z, r)$ plane for (a) jetd0, (b) jetd2, (c) jetd3 and (d) jetd4. The color scales range from 110 to $160 \mathrm{~dB}$, from blue to red. Black lines represent isocontours of OASPL from (a) 130 to $150 \mathrm{~dB}$, (b,c) 120 to $150 \mathrm{~dB}$ and (d) 110 to $130 \mathrm{~dB}$ with an increment of $2 \mathrm{~dB}$. 
steps. During the first step, a coherent structure is convected in the shear layer to the plate, where its impingement produces acoustic waves. During the second step, these waves propagate upstream to the nozzle, where they excite the shear layer, developping a new coherent structure and closing the feedback loop. Ho \& Nosseir [2] proposed a model to predict the feedback frequency. This model is built by considering the feedback period as the sum of two characteristic times. The first one is the time of convection of the flow structures from the nozzle to the plate and the second one is the time of propagation of the acoustic waves in the upstream direction. The feedback frequency $f$ can thus be predicted by

$$
f=\frac{N\left\langle u_{c}\right\rangle}{L\left(1+M_{c}\right)}
$$

where $N$ is an integer and $M_{c}=\left\langle u_{c}\right\rangle / c_{0}$ is the convection Mach number. The integer $N$ represents the order of the feedback mode and corresponds to the number of coherent structures between the nozzle and the plate. The convection velocities determined in section III.C are used to compute the feedback frequencies predicted by the model, given in table 2 for $N=3$ and $N=5$. These values of $N$ are chosen because they give frequencies close to those of tones observed in the spectra. Strouhal numbers between 0.39 and 0.44 are found for $N=3$. These values are in agreement with the tone Strouhal numbers of 0.41 in the spectra, which supports the existence of a feedback loop for all jets, at a frequency negligibly depending on the plate hole. Concerning jetd4, the hole is so large that the structures do not impinge on solid surfaces, creating a weak feedback loop and a tone of low intensity in the pressure spectra. For $N=5$, Strouhal numbers between 0.68 and 0.73 are found. These values are close of those between 0.60 and 0.68 found in the spectra of jetd0, jetd2 and jetd3, which indicates a second feedback loop.

\begin{tabular}{ccccc}
$\mathrm{N}$ & jetd0 & jetd2 & jetd3 & jetd4 \\
\hline 3 & 0.41 & 0.44 & 0.43 & 0.39 \\
5 & 0.68 & 0.73 & 0.72 & 0.65
\end{tabular}

Table 2 Feedback Strouhal numbers predicted by the model of Ho \& Nosseir [2] for $N=3$ and $N=5$.

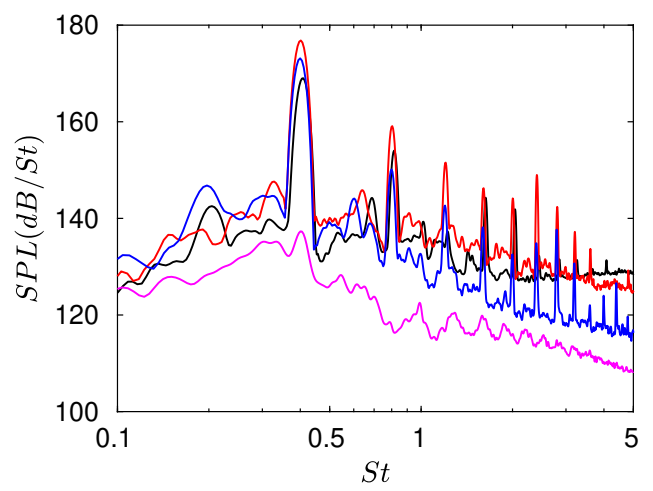

Fig. 10 Sound pressure levels (SPL) at $r=2 r_{0}$ and $z=0$ as a function of the Strouhal number $S t=f D / u_{j}$; — jetd0, 一 jetd2, 一 jetd3, - jetd4.

\section{F. Azimuthal structure of the jets}

For the four impinging jets, the pressure fields have been decomposed in their two first azimuthal modes. The contributions of these modes to the SPL at $r=2 r_{0}$ and $z=0$ are provided in figure 11 . For the no hole case, the most intense tones at $S t=0.41,0.82$ and 1.20 , are found for the axisymmetric mode $n_{\theta}=0$, which is in agreement with the vortex sheet model developed by Tam \& Ahuja [32] predicting an axisymmetric oscillation mode for subsonic impinging jets. For the sake of conciseness, the model is not detailed here. Moreover, the spectrum associated to the mode $n_{\theta}=1$ displays a peak at $S t=0.68$. This frequency verifies equation (1) for $N=5$, as can be seen in table 2 . This result suggests the establishment of an helical feedback mode. Such a mode is in agreement with the experimental 
study of Panickar \& Raman [33], revealing the coexistence of an axisymmetric and an helical instability mode for impinging jets at a Mach number higher than 0.89. For jetd2 and jetd 3 represented in figures 11 (b) and 11 (c), the spectra associated to the azimuthal modes are very similar to those for jetd0. Indeed, the dominant tones are linked to the axisymmetric mode whereas the helical mode presents weaker tones around $S t=0.65$ and $S t=0.60$ for jetd2 and jetd3, respectively. These Strouhal numbers are slighly lower than those predicted by relation (1) for $N=5$. For jetd 4 in figure 11(d), the tone around $S t=0.41$ can be related to the axisymmetric mode whereas no significative frequency emerges in the spectrum of the helical mode.

(a)

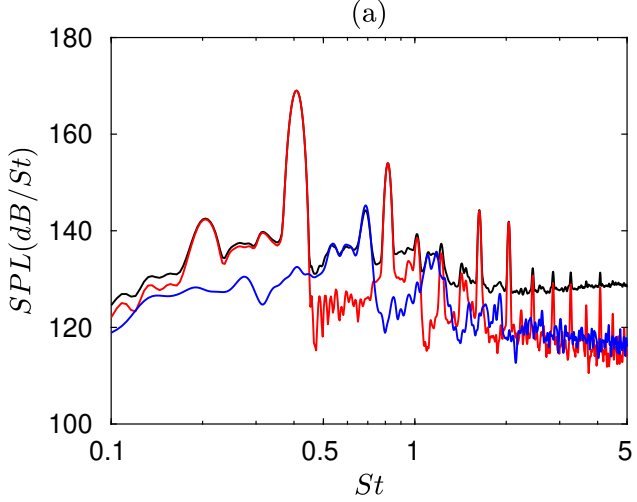

(c)

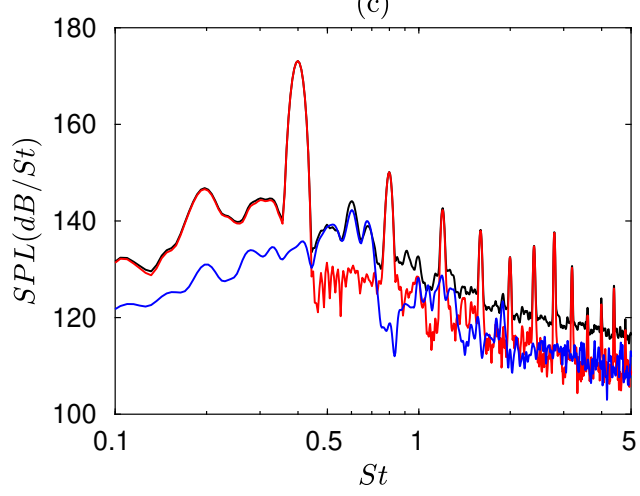

(b)

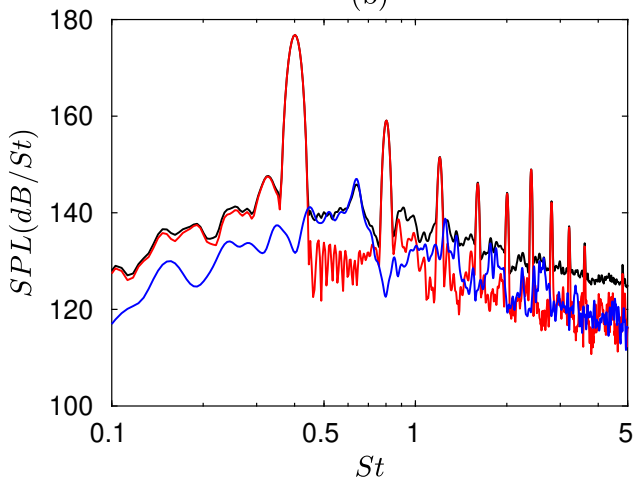

(d)

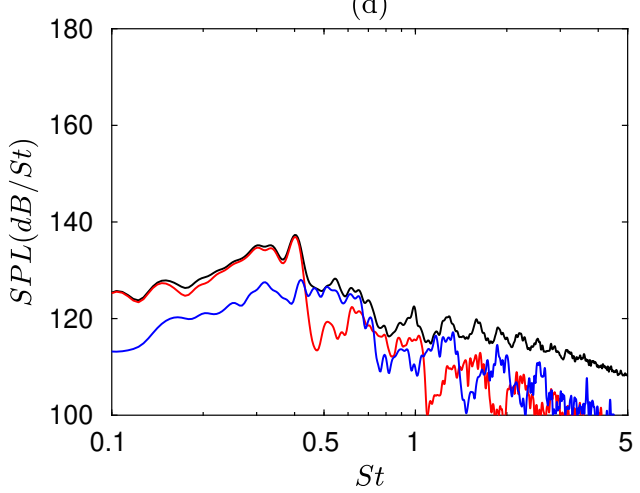

Fig. 11 Azimuthal decomposition of the sound pressure levels (SPL) at $r=2 r_{0}$ and $z=0$ for (a) jetd0, (b) jetd2, (c) jetd3 and (d) jetd4; - full SPL, azimuthal modes $-n_{\theta}=0,-n_{\theta}=1$.

\section{G. Fourier decomposition of the pressure field}

The axisymmetric and helical modes of the near pressure fields of the jets are decomposed using a Fast Fourier Transform in the time domain following the method applied to ideally expanded supersonic impinging jets by Gojon et al. [7]. The amplitude fields obtained for the axisymmetric mode at the dominant tone frequency of $S t=0.41$ are displayed in figure 12. For jetd0, three spots of significant amplitude are visible in the jet in figure 12(a). They are characteristic of a standing wave with three nodes establishing between the nozzle and the plate. Such a standing wave has been described by Panda et al. [34] for screeching jets. It is formed by the superposition of hydrodynamic waves propagating downstream and acoustic waves travelling upstream. The hydrodynamic waves involved in the standing wave pattern are linked to the turbulent structures convected in the jet flow. In this way, the three nodes correspond to three vortical structures between the nozzle and the plate, which is in agreement with the value of $N=3$ of the feedback model of Ho \& Nosseir [2] determined in section III.E. A similar standing wave structure is found in figures [12 b) and 12 (c) for jetd2 and jetd3. For these two jets, four lobes are visible in the amplitude fields. Three of them are located at the same axial position as for jetd0. The last node is located near the hole and is linked to the coherent structures passing periodically through the hole. For jetd4, in figure 12 (d), lobes are visible in the jet upstream of the plate, but they are not as marked as for the other jets. A zone of high amplitude is also observed in the jet from $z=6 r_{0}$, suggesting another 
sound generation mechanism at this frequency, such as the scattering of the aerodynamic pressure by the hole edges. This mechanism might coexist with the feedback loops.
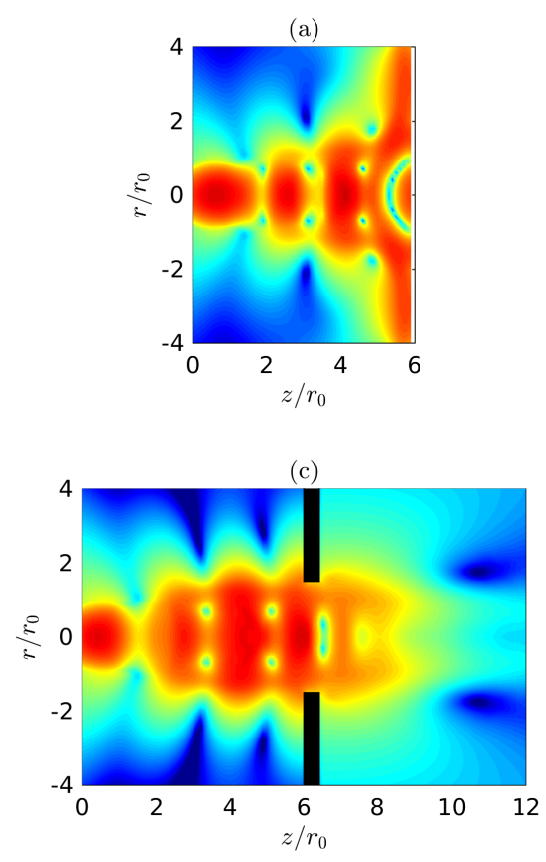
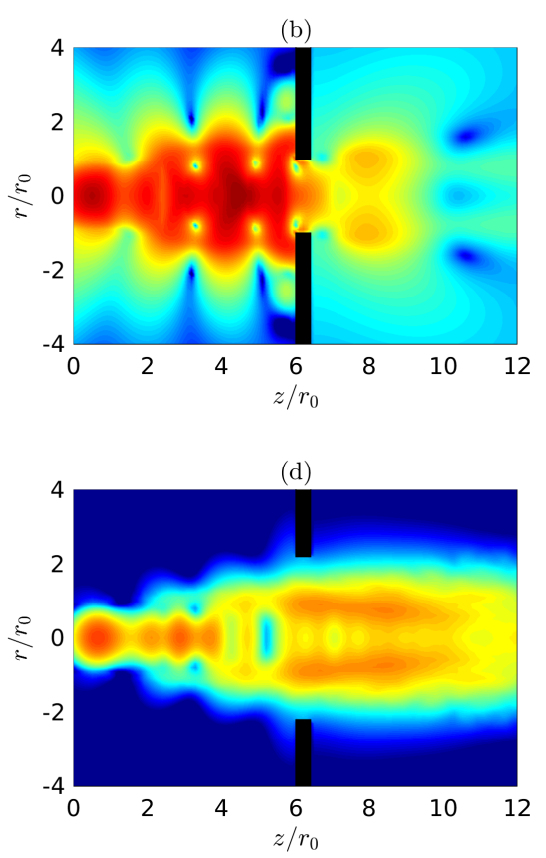

Fig. 12 Sound pressure levels for the axisymmetric mode at the dominant tonal frequency $S t=0.41$ for (a) jetd0, (b) jetd2, (c) jetd3 and (d) jetd4. The color scales range from (a, b, c) 150 to $200 \mathrm{~dB} / \mathrm{St}$ and (d) 140 to 170 $\mathrm{dB} / \mathrm{St}$, from blue to red.

The sound pressure levels of the helical mode at its dominant tonal frequency are displayed in figure 13 for jetd0, jetd 2 and jetd3. The helical mode is characterized by a zero amplitude on the jet axis. For these three jets, five lobes of amplitude are found upstream of the plate, agreeing with the value of $N=5$ of the feedback model of Ho \& Nosseir [2]. This result confirms thus the existence of an helical feedback mode predicted by Panickar \& Raman [33]. For the plates with a hole, downstream of the plate, the sound pressure levels are the highest in the mixing layers of the jets.
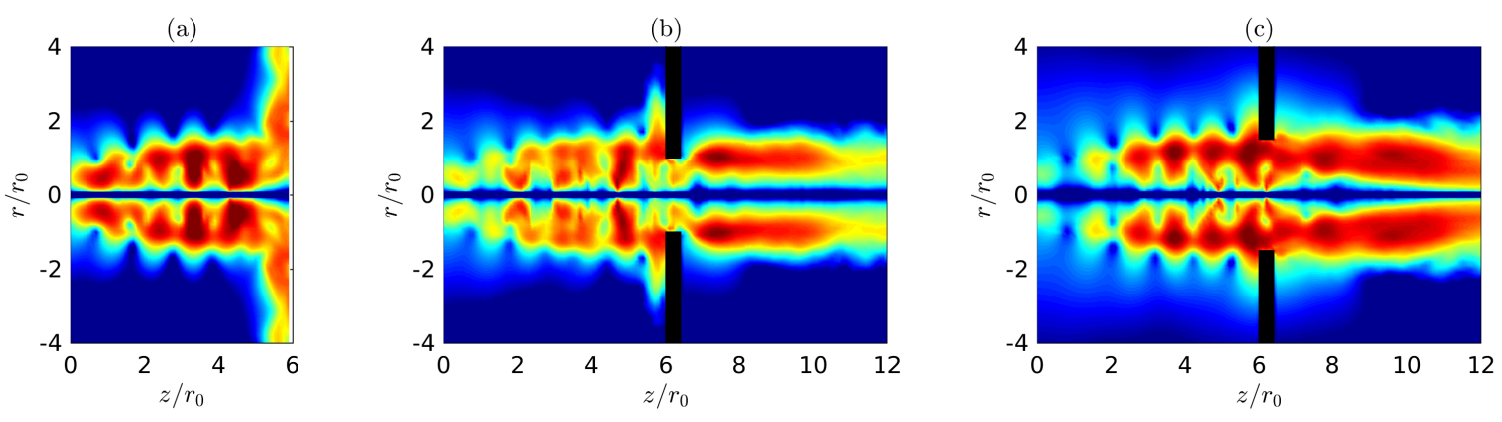

Fig. 13 Sound pressure levels for the first helical mode for (a) jetd0 at $S t=0.69$, (b) jetd2 at $S t=0.65$ and (c) jetd3 at $S t=0.60$. The color scales range from 140 to $165 \mathrm{~dB} / \mathrm{St}$, from blue to red. 


\section{H. Acoustic field downstream of the plate}

For jetd 2 and jetd3, an intense tonal radiation is visible downstream of the plate in figure 3 . Such a radiation was observed by Umeda \& Ishii [24] for a supersonic jet impinging on a plate with a hole. These authors suggested that it is caused by the periodic passing of vortical structures through the hole leading to flow-rate fluctuations through the hole. Unfortunately, this hypothesis was not confirmed yet by measurement of the flow rate. Therefore, the mass flow rate $m(t)$ through the hole is examined for jetd 2 and jetd 3 . It is defined by

$$
m(t)=\int_{0}^{2 \pi} \int_{0}^{d / 2} \rho(r, \theta, z=L, t) u_{z}(r, \theta, z=L, t) r d r d \theta
$$

where $\rho$ is the density. The time variations of the flow rate, normalized by the mass flow rate at the nozzle exit $m_{0}=\rho_{0} u_{j} \pi r_{0}^{2}$, are presented in figure 14. For jetd2, the flow rate is lower than $m_{0}$ because of the development of a wall jet, diverting a significant part of the flow in the radial direction. For jetd3, the flow rate is higher than $m_{0}$ because no wall jet develops leading to an increase of the flow rate due to the jet entrainment. In both cases, the flow rates oscillate at the feedback frequency. In aeroacoustics, the flow rate fluctuations are usually associated to a monopole radiation. In order to study the effects of these fluctuations on the acoustic field, they are modeled by a monopole source located at $r=0$ and $z=L$ generating the same flow rate in the downstream direction. The fluctuations of pressure created by this source at a point $M$ are given by

$$
p^{\prime}(M, t)=\frac{1}{2 \pi R} \frac{d m}{d t}\left(t-\frac{R}{c_{0}}\right)
$$

with $R$ the distance between the source and point $M$ [35]. The pressure fields thus obtained are presented in figures 15$](\mathrm{b}, \mathrm{d})$ for jetd 2 and jetd3. In both cases, the monopole source radiates spherical periodical waves, yielding acoustic fields very similar to the LES results displayed on figures $15(\mathrm{a}, \mathrm{c})$. This agreement between the model and the LES confirms the hypothesis of Umeda \& Ishii [24] of a monopole radiation downstream of the plate.

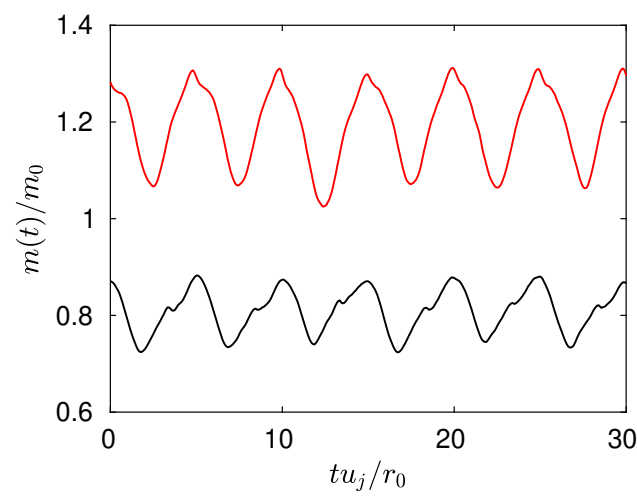

Fig. 14 Time variations of the mass flow rate through the hole normalized by the mass flow rate at the nozzle exit $m_{0}:-$ jetd2, - jetd3. 

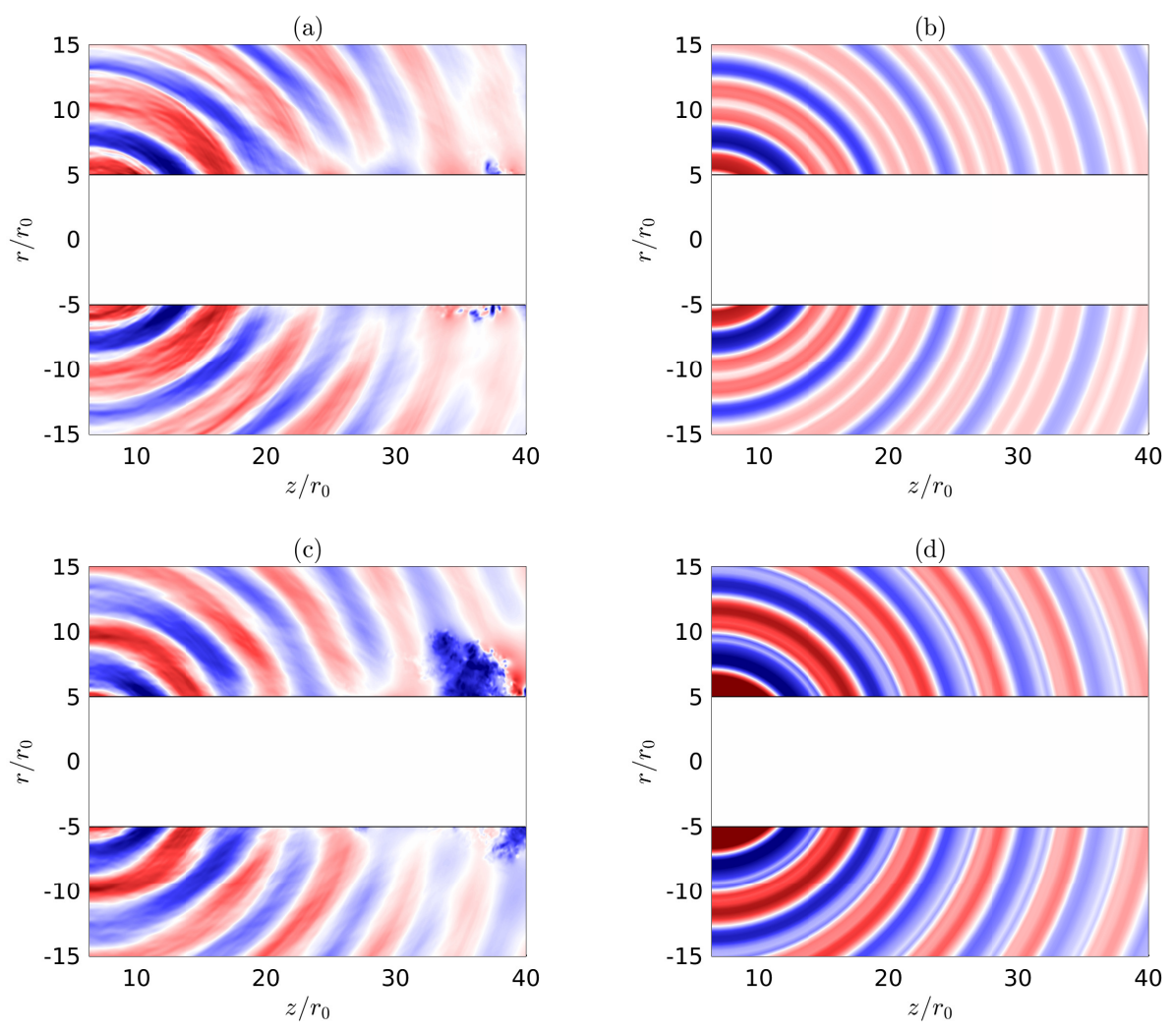

Fig. 15 Pressure fields downstream of the plate obtained (left) by LES and (right) by a monopole source of equivalent flow rate for (top) jetd2 and (bottom) jetd3. 


\section{Conclusion}

In the present study, the effects of the hole on the flow and acoustic fields of jets impinging on a plate with a hole have been investigated by simulations of jets at a Mach number of 0.9 , namely one jet impinging on a plate without a hole and three jets impinging on a plate with a hole diameter of $2 r_{0}, 3 r_{0}$ and $4.4 r_{0}$. For the plate with no hole, the flow and pressure fields snapshots show an acoustic tonal radiation. The pressure spectra near the nozzle also reveal several tone frequencies, which are explained by a feedback mechanism between the nozzle and the plate. These frequencies are in agreement with the feedback model of Ho \& Nosseir [2]. For the hole diameters of $2 r_{0}$ and $3 r_{0}$, the same feedback mechanism as for the previous case establishes. A tonal radiation is also observed downstream of the plate. The pressure spectra near the nozzle present tones at the same frequency as for the case without a hole. The hole has therefore little effects on the feedback frequency. For the hole diameter of $4.4 r_{0}$, the pressure spectra exhibit a tone of low amplitude at the feedback frequency, indicating a weak feedback loop. A Fourier decomposition at the dominant tonal frequency has been applied to the pressure fields of the axisymmetric and helical mode of the jets. For all jets, the decomposition highlights a dominant axisymmetric mode and a standing-wave structure between the plate and the nozzle. Moreover, except for the largest hole, this dominant axisymmetric mode coexist with a helical mode of lower intensity. Finally, for $d=2 r_{0}$ and $3 r_{0}$, the acoustic field downstream of the plate is modeled by a monopole source with the same flow rate as the jet flow rate through the hole. The prediction of the model is in agreement with the results of the LES, which validates the hypothesis of previous investigators of a monopole source downstream of the plate [24]. However, the sound generation mechanism downstream of the plate with the largest hole remain unclear, which motivates further investigations.

\section{Acknowledgments}

This work was financed by ArianeGroup and the DGA (Direction Générale de l'Armement). It was granted access to the HPC resources of FLMSN (Fédération Lyonnaise de Modélisation et Sciences Numériques), partner of EQUIPEX EQUIP@MESO, and of the resources of IDRIS (Institut du Développement et des Ressources en Informatique Scientifique) under the allocation 2019-2a0204 made by GENCI (Grand Equipement National de Calcul Intensif). It was performed within the framework of the Labex CeLyA of Universite de Lyon, operated by the French National Research Agency (grant no. ANR-10-LABX-0060/ANR-16-IDEX-0005).

\section{References}

[1] Powell, A., "On edge tones and associated phenomena," Acta Acustica United with Acustica, Vol. 3, No. 4, 1953 , pp. $233-243$.

[2] Ho, C.-M., and Nosseir, N. S., "Dynamics of an impinging jet. Part 1. The feedback phenomenon," Journal of Fluid Mechanics, Vol. 105, 1981, pp. 119-142.

[3] Nosseir, N. S., and Ho, C.-M., "Dynamics of an impinging jet. Part 2. The noise generation,” Journal of Fluid Mechanics, Vol. 116, 1982, pp. 379-391.

[4] Neuwerth, G., "Acoustic feedback of a subsonic and supersonic free jet which impinges on an obstacle," NASA Technical Translation No. F-15719, 1974.

[5] Preisser, J. S., "Fluctuating surface pressure and acoustic radiation for subsonic normal jet impingement," NASA Technical Paper 1361, 1979.

[6] Norum, T. D., "Supersonic rectangular jet impingement noise experiments," AIAA Journal, Vol. 29, No. 7, 1991, pp. $1051-1057$.

[7] Gojon, R., Bogey, C., and Marsden, O., "Investigation of tone generation in ideally expanded supersonic planar impinging jets using large-eddy simulation," Journal of Fluid Mechanics, Vol. 808, 2016, pp. 90-115.

[8] Bogey, C., and Gojon, R., "Feedback loop and upwind-propagating waves in ideally expanded supersonic impinging round jets," Journal of Fluid Mechanics, Vol. 823, 2017, pp. 562-591.

[9] Henderson, B., Bridges, J., and Wernet, M., "An experimental study of the oscillatory flow structure of tone-producing supersonic impinging jets,” Journal of Fluid Mechanics, Vol. 542, 2005, pp. 115-137.

[10] Buchmann, N., Mitchell, D., Ingvorsen, K. M., Honnery, D., and Soria, J., "High spatial resolution imaging of a supersonic underexpanded jet impinging on a flat plate," 6th Australian Conference on Laser Diagnostics in Fluid Mechanics and Combustion, 2011. 
[11] Risborg, A., and Soria, J., "High-speed optical measurements of an underexpanded supersonic jet impinging on an inclined plate," 28th International Congress on High-Speed Imaging and Photonics, Vol. 7126, International Society for Optics and Photonics, 2009, p. 71261F.

[12] Mitchell, D. M., Honnery, D. R., and Soria, J., "The visualization of the acoustic feedback loop in impinging underexpanded supersonic jet flows using ultra-high frame rate schlieren," Journal of Visualization, Vol. 15, No. 4, 2012, pp. $333-341$.

[13] Gojon, R., and Bogey, C., "Flow structure oscillations and tone production in underexpanded impinging round jets," AIAA Journal, Vol. 55, No. 6, 2017, pp. 1792-1805.

[14] Dauptain, A., M. Gicquel, L., and Moreau, S., "Large-eddy simulation of supersonic impinging jets," AIAA Journal, Vol. 50, No. 7, 2012, pp. 1560-1574.

[15] Tsutsumi, S., Takaki, R., Ikaida, H., and Terashima, K., "Numerical Aeroacoustics analysis of a scaled solid jet impinging on flat plate with exhaust hole," 30th International Symposium on Space Technology and Science, 2015.

[16] Troyes, J. N., Vuillot, F., Langenais, A., and Lambaré, H., "Coupled CFD-CAA Simulation of the Noise Generated by a Hot Supersonic Jet Impinging on a Flat Plate with Exhaust Hole,” AIAA Paper 2019-2752, 2019.

[17] Sondhauss, C., "Über die beim Ausströmen der Luft entstehenden Töne,” Annalen der Physik, Vol. 167, No. 2, 1854, pp. 214-240.

[18] Rayleigh, L., "The theory of sound, vols. I and II,”, 1945.

[19] Chanaud, R., and Powell, A., "Some experiments concerning the hole and ring tone," The Journal of the Acoustical Society of America, Vol. 37, No. 5, 1965, pp. 902-911.

[20] Vinoth, B., and Rathakrishnan, E., "Effect of impinging plate geometry on the self-excitation of subsonic impinging jets," Journal of Fluids and Structures, Vol. 27, No. 8, 2011, pp. 1238-1251.

[21] Langthjem, M. A., and Nakano, M., "The jet hole-tone oscillation cycle subjected to acoustic excitation: a numerical study based on an axisymmetric vortex method," The Proceedings of the International Conference on Jets, Wakes and Separated Flows 2005, The Japan Society of Mechanical Engineers, 2005, pp. 745-750.

[22] Matsuura, K., and Nakano, M., "A throttling mechanism sustaining a hole tone feedback system at very low Mach numbers," Journal of Fluid Mechanics, Vol. 710, 2012, pp. 569-605.

[23] Umeda, Y., Maeda, H., and Ishii, R., "Hole tone generated from almost choked to highly choked jets," AIAA Journal, Vol. 26, No. 9, 1988, pp. 1036-1043.

[24] Umeda, Y., and Ishii, R., "Hole tone generation from highly choked jets," The Journal of the Acoustical Society of America, Vol. 94, No. 2, 1993, pp. 1058-1066.

[25] Bogey, C., "Grid sensitivity of flow field and noise of high-Reynolds-number jets computed by large-eddy simulation," International Journal of Aeroacoustics, Vol. 17, No. 4-5, 2018, pp. 399-424.

[26] Berland, J., Bogey, C., Marsden, O., and Bailly, C., "High-order, low dispersive and low dissipative explicit schemes for multiple-scale and boundary problems," Journal of Computational Physics, Vol. 224, No. 2, 2007, pp. 637-662.

[27] Bogey, C., and Bailly, C., "A family of low dispersive and low dissipative explicit schemes for flow and noise computations," Journal of Computational Physics, Vol. 194, No. 1, 2004, pp. 194-214.

[28] Tam, C., and Dong, Z., "Radiation and outflow boundary conditions for direct computation of acoustic and flow disturbances in a non uniform mean flow," Journal of Computational Acoustics, Vol. 4, No. 02, 1996, pp. 175-201.

[29] Bogey, C., and Bailly, C., "Three-dimensional non-reflective boundary conditions for acoustic simulations: far-field formulation and validation test cases," Acta Acustica united with Acustica, Vol. 88, No. 4, 2002, pp. 463-471.

[30] Mohseni, K., and Colonius, T., "Numerical treatment of polar coordinate singularities," Journal of Computational Physics, Vol. 157, No. 2, 2000, pp. 787-795.

[31] Bogey, C., De Cacqueray, N., and Bailly, C., "Finite differences for coarse azimuthal discretization and for reduction of effective resolution near origin of cylindrical flow equations," Journal of Computational Physics, Vol. 230, No. 4, 2011, pp. 1134-1146. 
[32] Tam, C., and Ahuja, K., "Theoretical model of discrete tone generation by impinging jets," Journal of Fluid Mechanics, Vol. 214, 1990, pp. 67-87.

[33] Panickar, P., and Raman, G., "Criteria for the existence of helical instabilities in subsonic impinging jets," Physics of Fluids, Vol. 19, No. 10, 2007, pp. 103-106.

[34] Panda, J., Raman, G., and Zaman, K., "Underexpanded screeching jets from circular, rectangular and elliptic nozzles,” AIAA Paper 1997-1623, 1997.

[35] Russell, D. A., Titlow, J. P., and Bemmen, Y., "Acoustic monopoles, dipoles, and quadrupoles: An experiment revisited," American Journal of Physics, Vol. 67, No. 8, 1999, pp. 660-664. 